\title{
The Association of Systemic Inflammatory Markers With Indicators of Stress and Cardiac Necrosis in Patients Undergoing Aortic Valve Replacement and Revascularization Surgeries
}

\author{
Andrea SARACEVIC ${ }^{1}$, Igor MEDVED ${ }^{2}$, Snjezana HRABRIC VLAH ${ }^{3}$, Ana KOZMAR ${ }^{4}$, \\ Lidija BILIC-ZULLE ${ }^{3,5}$, Ana-Maria SIMUNDIC ${ }^{1,6}$ \\ ${ }^{1}$ Department of Medical Laboratory Diagnostics, University Hospital "Sveti Duh", Zagreb, Croatia, \\ ${ }^{2}$ Department of Cardiac Surgery, Clinical Hospital Centre Rijeka, Rijeka, Croatia, ${ }^{3}$ Clinical \\ Department of Laboratory Diagnostics, Clinical Hospital Centre Rijeka, Rijeka, Croatia, \\ ${ }^{4}$ Department of Laboratory Diagnostics, University Hospital Centre Zagreb, Zagreb, \\ Croatia, ${ }^{5}$ Department of Medical Informatics, Rijeka University Faculty of Medicine, Rijeka, \\ Croatia, ${ }^{6}$ Faculty of Pharmacy and Biochemistry, University of Zagreb, Zagreb, Croatia
}

Received June 27, 2019

Accepted January 7, 2020

Epub Ahead of Print March 23, 2020

\section{Summary}

The aim was to investigate: changes of inflammatory, stress and cardiac response in patients undergoing open heart surgeries up to five days after the procedure; the association between inflammatory, stress and cardiac response and whether changes in a certain marker can predict short-term patient outcome. Ninety patients were divided into three groups, 30 participants each (on-pump, off-pump revascularization and valve replacement group). The following markers were measured: complete blood count, CRP, IL-6, IL-10, leptin, resistin, monocyte chemoattractant protein-1 (MCP-1), cortisol, CK and hsTnT in 5 points. Resistin increased in all three groups. Lower IL-10 levels were found after the surgery and higher levels of leptin and MCP1 in the off-pump than in the on-pump group. Off-pump group had higher values of IL-6, IL-10, leptin, resistin and MCP- 1 and lower levels of CK and $h s T n T 24$ after surgery than the on-pump group. We found significant correlation between MCP-1 and resistin. The difference between resistin at time points 2 and 3 significantly predicted transfusion needs; while the difference between CRP and resistin before and at the end of the surgery together with the difference between leukocytes at the end and 24 hours after the surgery predicted the use of inotropic agents/vasopressors. Cardiac surgeries cause an increase of inflammatory, stress and cardiac markers. Only resistin correlated with MCP-1 which confirms the link between resistin secreted form infiltrated macrophages and enhanced release of MCP-1.

\section{Key words}

Cardiopulmonary bypass • Coronary artery bypass grafts surgery - Heart valve surgery - Inflammation - systemic (incl. cells, mediators) • Ischemia/reperfusion

\section{Corresponding author}

Andrea Saracevic, Department of Medical Laboratory Diagnostics, University Hospital "Sveti Duh", Sveti Duh 64, Zagreb, Croatia. E-mail: andrea.saracevic@gmail.com

\section{Introduction}

Cardiovascular diseases (CVD) are a major health problem in the modern society. Millions of new CVD cases are diagnosed each year throughout the globe. Only in Europe, CVD caused around 4 million deaths in 2017 which accounted for $45 \%$ of all deaths (European Cardiovascular Disease Statistics 2017). For these reasons open heart surgeries are the most common operating techniques worldwide and out of them, the most frequent ones are coronary artery bypass surgery (with and without the use of cardiopulmonary bypass $(\mathrm{CPB}))$ and heart valve replacement.

Although being inevitable for treating seriously affected heart, open heart surgery triggers certain adverse pathophysiologic changes in the organism by inducing

PHYSIOLOGICAL RESEARCH • ISSN 1802-9973 (online) 
systemic stress and inflammatory responses. Such response is accompanied by activation of the coagulation and immune system, triggering the hypothalamicpituitary-adrenal axis which leads to a substantial release of stress hormones and leukocyte recruitment. Consequently, numerous pro- and anti-inflammatory cytokines and acute phase reactants are produced which altogether leads to a complex pleiotropic reaction (Castellheim et al. 2008, Onorati et al. 2010, Gibbison et al. 2015, Kawahito et al. 2000). Alongside the global processes in the organism, micro-environmental changes also occur within the operated heart. Myocardial ischemia causes adverse changes within the cardiomyocytes that can lead to cellular necrosis and stimulate monocyte recruitment that differentiate into cardiac macrophages. These cardiac macrophages further release factors that stimulate cell survival, regeneration and regulate the inflammation (Frangogiannis 2015).

Depending on the operating technique and the utilization of $\mathrm{CPB}$, the immune and stress response within the body and the operated heart varies. The use of extracorporeal circulation leads to a strong response due to the exposure of the blood to artificial surfaces of the $\mathrm{CPB}$ and causing global myocardial ischemia-reperfusion injury. Operating on the beating heart theoretically, leads to a smaller response due to eliminating the above mentioned global negative stimuli and causing only local, smaller ischemia-reperfusion injuries of the heart around the coronary anastomoses.

Cytokines and adipokines from peripheral, as well as epicardial adipose tissue act as cardiac function modulators (Matloch et al. 2016). Besides modulating heart function, these molecules play pivotal roles in inflammatory cascade reactions. Investigations have shown increase of interleukin 6 (IL-6), interleukin 10 (IL-10) monocyte chemoattractant protein-1 (MCP-1) concentrations during the perioperative period of cardiac surgery. Some studies showed that the increase of IL-10 during on-pump coronary artery bypass grafting (CABG) is significantly higher than in off-pump revascularization (OPCAB), while there are inconsistent results regarding the difference in IL- 6 and MCP-1 between on-pump and off-pump procedures (Castellheim et al. 2008, Biglioli et al. 2003, Wehlin et al. 2005). A recent meta-analysis showed that there is no statistically significant difference in perioperative IL- 6 concentrations between the CABG and OPCAB, while the IL-10 concentration 12 hours after surgery was significantly lower in OPCAB group compared to CABG group (Meng et al. 2017).
Additionally, one study found that the concentrations of IL-6 and MCP-1 were higher in patients undergoing valve replacements than in patients undergoing CABG (Kawahito et al. 2000).

Leptin is an adipokine secreted from the adipose tissue but also from certain peripheral tissues including the cardiomyocytes where it regulates contractility, cell apoptosis and metabolism (Feijóo-Bandín et al. 2015). The changes in leptin concentration have been investigated not only during cardiac surgery, but also in different cardiac conditions, i.e. heart failure (Modan-Moses et al. 2001, Hoda et al. 2006, Laurikka et al. 2014, Cundrle et al. 2018). During cardiac surgery leptin initially shows a decrease in concentration, followed by an increase after the surgery that lasts up to 48 hours (Modan-Moses et al. 2001, Hoda et al. 2006, Laurikka et al. 2014).

In addition to leptin, resistin is another adipokine that plays an important role in immune response. Similarly, to leptin, resistin showed a short transient decrease in concentration at the beginning of the surgery, slowly rising to either initial values or, as some authors have shown, even up to 2.5 -fold or higher than the baseline values (Laurikka et al. 2014, Németh et al. 2014, Kremen et al. 2006).

To the best of our knowledge, only one study has so far investigated the time course of resistin in patients undergoing aortic valve replacement, unfortunately on a very small number of participants $(\mathrm{N}=5)$; while no one has compared its course with other types of open-heart surgery. Since resistin in humans in mostly secreted from macrophages and it upregulates the expression of proinflammatory cytokines (TNF- $\alpha$, IL- 6 , IL-12 and MCP-1), it is possible that its concentration might predict the development of complications and patient outcome after open heart surgery.

We hypothesized patients attached to CPB have stronger systemic response to surgery and thus have higher concentrations of inflammatory, stress ad cardiac necrosis markers during the perioperative period than patients undergoing surgery without CPB. Additionally, we hypothesized that the inflammatory response correlates with the magnitude of stress and myocardial necrosis extent; and finally that the intensity of the inflammation could predict patient outcome and the occurrence of postoperative complications.

Therefore, the aim of our study was to investigate changes of systemic inflammatory response, changes of stress and cardiac markers in patients undergoing three most common open heart surgeries - 
coronary revascularization with and without the use of $\mathrm{CPB}$ and aortic valve replacement up to five days after the surgical procedure. In addition, we aimed to investigate the association between systemic inflammatory response and stress and cardiac necrosis markers and to examine whether intensity of changes in a certain marker can predict short-term patient outcome.

\section{Materials}

\section{Study design}

The study was conducted at the Clinical Hospital Centre Rijeka in collaboration with the Department of Medical Laboratory Diagnostics, University Hospital "Sveti Duh" in the period between July 2015 and July 2017. The research was conducted in accordance with the Declaration of Helsinki after it was approved by the Institution's Ethical Committee. All patients signed an informed consent.

Patients with multi-vessel coronary artery disease (CAD) and/or stenosis of the left main coronary artery undergoing coronary artery bypass surgery and elective patients undergoing aortic valve replacement surgery were recruited into the study. A total of 90 patients were divided into three groups each consisting of 30 participants - on-pump (CABG), off-pump (OPCAB) revascularization and valve replacement (AVRS) group. The exclusion criteria were: urgent patients, concomitant surgery, and patients who had to undergo a change in the operation plan during the operation (conversion from off-pump to on-pump revascularization).

\section{Surgical techniques}

All surgical access was made through median sternotomy and pericardiotomy.

Systemic heparinisation was used in all patients, with a dose of $150 \mathrm{IU} / \mathrm{kg}$ for off-pump group to reach activated clotting time (ACT) of 300 seconds, and a dose of $300 \mathrm{IU} / \mathrm{kg}$ for patients operated with the use of cardiopulmonary bypass to reach ACT of 480 seconds. Heparine reversal was made with protamine at the end of the procedure.

Off-pump revascularization was made in normothermia, while on-pump revascularization and aortic valve replacement were made in moderate hypothermia and with the use of extracorporeal circulation.

Extracorporeal cannulation setting for both on-pump revascularization and aortic valve replacement group was as follows: aortic cannula to distal ascending aorta, two-stage venous cannula through the right atrial appendage into the right atrium. Left ventricular vent through superior right pulmonary vein via left atrium to the left ventricle. Warm blood cardioplegia - Calafiore, was administered antegrade (cardioplegia catheter in the ascending aorta) and retrograde (retrograde cardioplegia catheter through the right atrium to coronary sinus).

\section{Aortic valve replacement technique}

After commencement of cardiopulmonary bypass, aortic cross-clamping and administration of cardioplegia by protocol followed. Approach to aortic valve was through transverse aortotomy. Excision of calcified aortic valve was made with aortic annulus decalcification. Single sutures were placed in the aortic annulus and aortic valve bioprosthesis was implanted. Aortotomy was closed by a continuous suture while de-airing of the heart and reperfusion were made. Weaning from cardiopulmonary bypass followed after acceptable hemodynamic values were achieved.

\section{On-pump Revascularization Group}

Internal mammary artery and great saphenous vein were simultaneously harvested for coronary artery bypass grafting. Systemic heparinisation by protocol and cardiopulmonary bypass with moderate hypotermia were established in a manner previously described. After aortic cross-clamping, distal coronary anastomoses were created. During rewarming and retrograde reperfusion of the heart, proximal coronary anastomoses to ascending aorta were made.

\section{Off-pump Revascularization Group}

Internal mammary artery and great saphenous

vein were simultaneously harvested. Systemic heparinisation by protocol for off-pump was given. Coronary anastomoses were performed with the use of vacuum-stabilizers on a beating heart and with the use of intracoronary shunts. Distal coronary anastomoses were created first, and then proximal coronary anastomoses to ascending aorta were made with the use of partial aortic cross-clamp.

After all cardiac procedures, protamine was administered to reverse effects of systemic heparinization. A meticulous hemostasis was made and a temporary epicardial pacemaker was implanted. Two pericardial drainage tubes were inserted. Sternum was 
closed with 4 wire sutures in a figure-of-eight fashion and sternal wound was sutured.

Blood samples for the study were obtained from coronary sinus after cessation of cardiopulmonary bypass before administration of protamine.

An $18 \mathrm{G}$ indwelling venous cannula and a non-heparinised syringe were used. The punctured site was closed with a single nonapsorbable monofilament suture.

\section{Methods}

Blood samples were taken from each patient to measure: complete blood count (CBC), C-reactive protein (CRP), IL-6 and IL-10, leptin, resistin, MCP-1, cortisol, creatine kinase $(\mathrm{CK})$ and high sensitive troponin $\mathrm{T}$ (hsTnT). Blood sampling was done according to the scheme presented in Table 1.

Table 1. Blood sampling scheme

\begin{tabular}{|c|c|c|c|}
\hline Sample No. & Time point & Sample Type & Analyte \\
\hline 1 & before the surgery & venous blood & $\begin{array}{c}\text { Leukocytes, CRP, CK, } \\
\text { IL-6, IL-10, leptin, resistin, MCP-1, } \\
\text { hsTnT, cortisol }\end{array}$ \\
\hline \multirow[t]{2}{*}{2} & $\begin{array}{l}\text { immediately before the end of } \\
\text { the surgery }\end{array}$ & coronary sinus blood & $\begin{array}{l}\text { IL-6, IL-10, leptin, resistin, MCP-1, } \\
\text { hsTnT, cortisol }\end{array}$ \\
\hline & & venous blood & Leukocytes, CRP, CK \\
\hline 3 & 24 hours after the surgery & venous blood & $\begin{array}{c}\text { Leukocytes, CRP, CK, IL-6, IL-10, leptin, } \\
\text { resistin, MCP-1, } \\
\text { hsTnT, cortisol }\end{array}$ \\
\hline 4 & 3 days after the surgery & venous blood & Leukocytes, CRP, CK \\
\hline 5 & 5 days after the surgery & venous blood & Leukocytes, CRP, CK \\
\hline
\end{tabular}

CRP- C-reactive protein; CK - creatine kinase; IL-6 - interleukin 6; IL-10 - interleukin 10, MCP-1 - monocyte chemoattractant protein-1, hsTnT- high sensitive troponin $\mathrm{T}$.

The tubes used for blood collection were, as follows:

1. Z Serum Sep Clot Activator serum tubes, $3.5 \mathrm{~mL}$ from Greiner Bio-one $\mathrm{GmbH}$, Kremsmunster, Austria for CRP, CK, hsTnT and cortisol;

2. K3EDTA tubes, $3 \mathrm{ml}$ from Becton Dickinson, Plymouth, United Kingdom for CBC, IL-6, IL-10, leptin, resistin, MCP-1.

Venous blood collected for CBC determination was processed immediately after blood sampling on the Cell-Dyn CD-1800 hematology analyzer (Abbott Diagnostics, Chicago, IL, USA). After the CBC determination, the EDTA tubes were centrifuged using Megafuge 1.0R (Kendro Laboratory products, Osterode, Germany) at $1000 \mathrm{x} \mathrm{g}$ for $15 \mathrm{~min}$. Following the centrifugation, $1 \mathrm{ml}$ of plasma was aliquoted and frozen at $-80{ }^{\circ} \mathrm{C}$ until analysis. Serum tubes for the rest of the markers were left to clot at room temperature for $30 \mathrm{~min}$, after which they were centrifuged with Megafuge 1.0R at
$1800 \mathrm{x} \mathrm{g}$ for $10 \mathrm{~min}$. Two aliquots of $500 \mu \mathrm{l}$ serum for each patient were frozen at $-80{ }^{\circ} \mathrm{C}$ until analysis.

CRP and CK were determined on AU400 platform (Beckman Coulter, Brea, California, USA), cortisol and hsTnT were measured on Cobas e411 analyzer (Roche Diagnostic, Mannheim, Germany). IL-6, IL-10, MCP-1, resistin and leptin were determined simultaneously by commercially available multiplex immunoassay kit (RnD systems, Minneapolis, MN; USA) on Luminex 200 platform (Luminex corporation, Austin, Texas, USA). Samples were tested according to the kit manufacturer's instructions.

The coefficients of variation of the investigated biomarkers are presented in Table 2. During the surgery the number of anastomoses performed and the length of the operation were recorded; while during the hospitalization the following clinical outcomes were monitored: time spent in the intensive unit, time to hospital discharge, duration of mechanical ventilation, 
Table 2. Coefficients of variation of the investigated biomarkers, as declared by the manufacturers.

\begin{tabular}{|c|c|c|c|c|c|}
\hline Analyte & Unit & Level & $\begin{array}{c}\text { Intra-assay CV } \\
(\%)\end{array}$ & Level & $\begin{array}{c}\text { Inter-assay } \\
\text { CV (\%) }\end{array}$ \\
\hline \multirow[t]{3}{*}{ C-reactive protein } & $\mathrm{mg} / \mathrm{l}$ & 6.44 & 0.80 & 6.44 & 3.12 \\
\hline & & 64.02 & 0.87 & 64.02 & 2.86 \\
\hline & & 137.70 & 0.74 & 137.70 & 2.63 \\
\hline \multirow[t]{3}{*}{ Creatine kinase } & $\mathrm{U} / 1$ & 99 & 2.37 & 99 & 4.55 \\
\hline & & 270 & 1.00 & 270 & 3.20 \\
\hline & & 8110 & 0.64 & 8110 & 3.28 \\
\hline \multirow[t]{2}{*}{ Cortisol } & $\mathrm{nmol} / \mathrm{l}$ & 363 & 1.4 & 363 & 1.6 \\
\hline & & 865 & 1.0 & 865 & 1.4 \\
\hline \multirow[t]{2}{*}{ High sensitive troponin $T$} & $\mathrm{ng} / \mathrm{l}$ & 25.6 & 2.1 & 25.6 & 3.6 \\
\hline & & 1819 & 1.5 & 1819 & 2.2 \\
\hline \multirow[t]{3}{*}{ Interleukin 6} & $\mathrm{pg} / \mathrm{ml}$ & 30.2 & 4.7 & 34 & 8.8 \\
\hline & & 180 & 4.3 & 189 & 6.9 \\
\hline & & 903 & 4.6 & 900 & 5.9 \\
\hline \multirow[t]{3}{*}{ Inerleukin 10} & $\mathrm{pg} / \mathrm{ml}$ & 10.4 & 5.2 & 12 & 8.3 \\
\hline & & 66 & 5.2 & 73 & 6.8 \\
\hline & & 320 & 6.5 & 333 & 7.2 \\
\hline Monocyte & $\mathrm{pg} / \mathrm{ml}$ & 58.7 & 3.8 & 66 & 7.6 \\
\hline chemoattractant & & 257 & 5.7 & 251 & 5.6 \\
\hline protein-1 & & 1067 & 6.4 & 1053 & 11.1 \\
\hline \multirow[t]{3}{*}{ Resistin } & $\mathrm{ng} / \mathrm{ml}$ & 0.108 & 6.8 & 0.129 & 9.2 \\
\hline & & 1.41 & 7.4 & 1.46 & 9.9 \\
\hline & & 3.13 & 9.6 & 3.28 & 8.7 \\
\hline \multirow[t]{3}{*}{ Leptin } & $\mathrm{ng} / \mathrm{ml}$ & 0.32 & 8.0 & 0.40 & 11.2 \\
\hline & & 2.75 & 4.6 & 2.88 & 7.2 \\
\hline & & 14.67 & 8.0 & 14.33 & 10.1 \\
\hline
\end{tabular}

blood loss in $\mathrm{ml}$, transfusion needs, use of ionotropic agents or vasopressors, electrocardiographic inotropic changes, atrial fibrillation, recurrent angina, postoperative myocardial infarction, stroke, kidney dysfunction, deep sternal bone infections, reoperation.

\section{Statistical analysis}

A power analysis was performed based on the published differences, standard deviations and reference change values for hsTnT, cortisol, IL-6, IL-10, resistin and leptin (Hoda et al. 2006, Frankenstein et al. 2011, Diegeler et al. 2000, Knudsen et al. 2008, Asgeirsson et al. 2011, Serrano-Martínez et al. 2003). The required sample size of 30 participants in each group based on these data was estimated for a significance level of 0.05 and a power of 0.8 .

All data sets have been tested for normality using the Kolmogorov Smirnov test. Since not all data sets were normally distributed, the results are presents as median (IQR).

For testing the differences between variables at different time points, the Kruskal-Wallis and ANOVA and Mann Whitney tests were used with post hoc analysis according to Conover and Student-Newman-Keuls test for all pairwise comparisons, as appropriate. The differences from the baseline values were tested using the by Wilcoxon and paired t-test, as appropriate. Additionally, the differences between categorical data 
Table 3. Patient characteristics

\begin{tabular}{|c|c|c|c|c|}
\hline Demographic characteristics & AVRS group & Off-pump group & On-pump group & P value \\
\hline$N$ & 30 & 30 & 30 & I \\
\hline Male gender, $N(\%)$ & $23(76.7 \%)$ & $20(66.7 \%)$ & $24(805)$ & $0.468^{*}$ \\
\hline Age $(\text { years })^{\dagger}$ & $67(21-80)$ & $67(53-78)$ & $69(52-77)$ & $0.5181^{* * *}$ \\
\hline Height $(\mathrm{cm})$ & $169(165-175)$ & $173(165-176)$ & $174(162-177)$ & $0.516^{* *}$ \\
\hline Weight (kg) & $83(74-92)$ & $84(75-92)$ & $84(80-90)$ & $0.928^{* *}$ \\
\hline \multirow[t]{3}{*}{ Hypertension, N/total } & $24 / 30$ & $26 / 30$ & $30 / 30$ & $1 v s .2: 0.731^{\$}$ \\
\hline & & & & $2 v s .3: 0.112^{\$}$ \\
\hline & & & & $1 v s .3: 0.024^{\$}$ \\
\hline Diabetes mellitus, $N /$ total & $9 / 30$ & $13 / 30$ & $19 / 30$ & $0.033^{*}$ \\
\hline Smoking, N/total & $4 / 30$ & $9 / 30$ & $7 / 30$ & $0.295^{*}$ \\
\hline Number of anastomoses & 1 & $3(3-5)$ & $5(3-5)$ & $0.018^{\#}$ \\
\hline Operation duration (min) & $180(160-200)$ & $180(150-200)$ & $180(180-225)$ & $0.123^{* *}$ \\
\hline $\begin{array}{l}\text { Time spent in the intensive unit } \\
\text { (hours) }\end{array}$ & $21(19-23)$ & $24(20-45)$ & $21(18-28)$ & $0.138 * * *$ \\
\hline $\begin{array}{l}\text { Time to hospital discharge } \\
\text { (days) }\end{array}$ & $7(5-7)$ & $6(6-8)$ & $7(6-8)$ & $0.450^{* * *}$ \\
\hline $\begin{array}{l}\text { Mechanical ventilation time } \\
\text { (hours) }\end{array}$ & $11(8-14)$ & $9(8-13)$ & $12(7-17)$ & $0.686^{* * *}$ \\
\hline $\begin{array}{l}\text { Blood loss through drainage } \\
\text { after surgery (ml) }\end{array}$ & $800(400-1300)$ & $760(470-1150)$ & $1050(800-1600)$ & $0.032 * *$ \\
\hline Transfusion needs, N/total & $5 / 30$ & $4 / 30$ & $10 / 30$ & 0.126 \\
\hline $\begin{array}{l}\text { Use of ionotropic agents or } \\
\text { vasopressors, N/total }\end{array}$ & $12 / 30$ & $2 / 30$ & $22 / 30$ & $<0.001$ \\
\hline $\begin{array}{l}\text { Electrocardiographic changes, } \\
\text { N/total }\end{array}$ & $2 / 30$ & $2 / 30$ & $5 / 30$ & $\begin{array}{l}1 v s .2: 1.000^{\S} \\
2 v s .3: 0.424^{\$} \\
1 v s .3: 0.424^{\$}\end{array}$ \\
\hline Atrial fibrillation, N/total & $0 / 30$ & $2 / 30$ & $6 / 30$ & $\begin{array}{l}1 v s .2: 0.492^{\$} \\
2 v s .3: 0.254^{\$} \\
1 v s .3: 0.024^{\$}\end{array}$ \\
\hline Recurrent angina, N/total & $0 / 30$ & $0 / 30$ & $0 / 30$ & 1.000 \\
\hline $\begin{array}{l}\text { Postoperative myocardial } \\
\text { infarction, N/total }\end{array}$ & $0 / 30$ & $0 / 30$ & $0 / 30$ & 1.000 \\
\hline Stroke & $0 / 30$ & $1 / 30$ & $0 / 30$ & 1.000 \\
\hline Kidney dysfunction, N/total & $0 / 30$ & $0 / 30$ & $0 / 30$ & 1.000 \\
\hline $\begin{array}{l}\text { Deep sternal bone infections, } \\
\text { N/total }\end{array}$ & $0 / 30$ & $0 / 30$ & $2 / 30$ & $\begin{array}{l}1 v s .2: 1.000^{\S} \\
2 v s .3: 0.492^{\$} \\
1 v s .3: 0.492^{\$}\end{array}$ \\
\hline Reoperation, N//total & $2 / 30$ & $0 / 30$ & $2 / 30$ & $\begin{array}{l}1 v s .2: 0.492^{\$} \\
2 v s .3: 0.492^{\$} \\
1 v s .3: 1.000^{\$}\end{array}$ \\
\hline
\end{tabular}

${ }^{*}$ chi square test, $* *$ ANOVA test, ${ }^{* * *}$ Kruskal-Wallis test, ${ }^{*}$ Mann-Whitney test, ${ }^{\$}$ Fisher's exact test, ${ }^{\dagger}$ age is presented as median (range) 
Table 4. Values of tested analytes at different time points for each group

\begin{tabular}{|c|c|c|c|c|c|}
\hline Analyte & $\begin{array}{l}\text { Time } \\
\text { point }\end{array}$ & AVRS group $\mathbf{N}=30$ & $\begin{array}{l}\text { Off-pump group, } \\
\qquad \mathbf{N}=\mathbf{3 0}\end{array}$ & On-pump group, $N=30$ & $P$ value \\
\hline \multirow{5}{*}{$\begin{array}{l}\text { Leukocytes } \\
\left(x 10^{9} / l\right)\end{array}$} & 1 & $5.7(4.9-6.9)$ & $6.8(5.6-8.4)$ & $6.6(5.3-8.6)$ & $0.477 * *$ \\
\hline & 2 & $8.8(6.5-11.0)$ & $6.4(4.8-8.6)$ & $7.1(5.1-9.3)$ & $0.029 *$ \\
\hline & 3 & $11.4(9.7-15.5)$ & $11.9(10.4-14.5)$ & $11.5(9.9-13.3)$ & $0.477^{*}$ \\
\hline & 4 & $10.8(10.1-12.6)$ & $9.8(8.2-12.4)$ & $10.9(8.1-12.2)$ & $0.252 *$ \\
\hline & 5 & $8.3(7.1-9.8)$ & $7.9(6.5-12.1)$ & $8.9(6.3-10.0)$ & $0.951 * *$ \\
\hline \multirow{5}{*}{$C R P(m g / l)$} & 1 & $1.0(1.0-2.2)$ & $1.6(1.0-3.9)$ & $1.5(1.0-3.1)$ & $0.297 * *$ \\
\hline & 2 & $1.0(1.0-1.6)$ & $1.4(1.0-2.9)$ & $1.0(1.0-1.9)$ & $0.104 * *$ \\
\hline & 3 & $64.0(49.9-90.1)$ & $96.0(67.7-117.6)$ & $90.7(64.1-115.3)$ & $0.012 * *$ \\
\hline & 4 & $142.9(81.7-174.8)$ & $144.2(102.9-178.4)$ & $157.9(123.2-189.1)$ & $0.303 *$ \\
\hline & 5 & $71.0(47.8-102.6)$ & $65.7(46.5-103.2)$ & $77.0(54.2-98.3)$ & $0.814^{* *}$ \\
\hline \multirow{3}{*}{$I L-6(p g / m l)$} & 1 & $7.1(7.1-7.1)$ & $41.9(7.1-63.9)$ & $7.1(7.1-7.1)$ & $0.001 * *$ \\
\hline & 2 & $47.6(16.1-178.5)$ & $134.1(49.8-338.0)$ & $100.7(48.5-303.8)$ & $0.092 * *$ \\
\hline & 3 & $65.1(45.4-136.6)$ & $470.5(162.1-663.8)$ & $74.6(46.5-110.4)$ & $<0.001 * *$ \\
\hline \multirow{3}{*}{$I L-10(\mathrm{pg} / \mathrm{ml})$} & 1 & $3.6(6.2-3.7)$ & $3.2(3.2-5.7)$ & $3.2(2.2-3.9)$ & $0.091 * *$ \\
\hline & 2 & $93.6(21.5-297.6)$ & $14.1(3.2-21.7)$ & 44.7 (11.0-125.7) & $<0.001 * *$ \\
\hline & 3 & $7.2(5.4-10.2)$ & $33.0(14.5-73.5)$ & $7.5(4.7-71.9)$ & $<0.001 * *$ \\
\hline \multirow{3}{*}{$\begin{array}{l}\text { Leptin } \\
(n g / m l)\end{array}$} & 1 & $26.3(10.4-43.7)$ & $33.6(15.5-54.9)$ & $15.4(9.6-23.2)$ & $0.030 * *$ \\
\hline & 2 & $16.9(5.5-26.3)$ & $26.8(11.4-46.1)$ & $9.7(6.4-14.4)$ & $0.006^{* *}$ \\
\hline & 3 & $24.6(9.4-39.8)$ & $39.9(19.9-61.8)$ & $14.2(6.3-21.9)$ & $0.002 * *$ \\
\hline \multirow{3}{*}{$\begin{array}{l}\text { Resistin } \\
(n g / m l)\end{array}$} & 1 & $7.0(5.7-10.3)$ & $19.1(9.9-24.2)$ & $10.2(7.7-14.9)$ & $<0.001 * *$ \\
\hline & 2 & $10.2(7.7-14.9)$ & $18.4(9.3-21.8)$ & $11.7(7.6-20.8)$ & $0.123 * *$ \\
\hline & 3 & $17.4(12.9-22.9)$ & $34.3(26.4-38.7)$ & $19.2(13.6-33.9)$ & $<0.001 * *$ \\
\hline \multirow{3}{*}{$\begin{array}{l}M C P-1 \\
(p g / m l)\end{array}$} & 1 & $119.9(98.1-152.4)$ & $519.6(188.2-790.3)$ & $135.6(102.0-188.7)$ & $<0.001 * *$ \\
\hline & 2 & $221.4(163.6-496.0)$ & $612.7(262.3-785.7)$ & 325.9 (188.9-640.9) & $0.045^{* *}$ \\
\hline & 3 & $107.9(85.5-137.3)$ & $480.5(165.5-570.8)$ & $135.6(102.0-188.7)$ & $<0.001 * *$ \\
\hline \multirow{5}{*}{$C K(U / l)$} & 1 & $41(26-53)$ & $35(25-60)$ & $65(30-89)$ & $0.081 * *$ \\
\hline & 2 & $127(78-190)$ & $39(28-57)$ & $120(88-188)$ & $<0.001 * *$ \\
\hline & 3 & $363(220-573)$ & $222(170-441)$ & $522(337-731)$ & $0.004 * *$ \\
\hline & 4 & $176(92-338)$ & $158(107-427)$ & $254(141-438)$ & $0.336^{* *}$ \\
\hline & 5 & $60(47-122)$ & $67(45-133)$ & $79(55-165)$ & $0.609 * *$ \\
\hline \multirow{3}{*}{$h s \operatorname{Tn} T(n g / l)$} & 1 & $17(13-25)$ & $14(8-22)$ & $11(9-23)$ & $0.277 * *$ \\
\hline & 2 & $150(105-226)$ & $94(47-282)$ & $97(53-162)$ & $0.107 * *$ \\
\hline & 3 & $331(253-433)$ & $136(79-182)$ & $243(166-410)$ & $<0.001 * *$ \\
\hline \multirow{3}{*}{$\begin{array}{l}\text { Cortisol } \\
\text { (nmol/l) }\end{array}$} & 1 & $390.2(245.8-444.6)$ & $392.2(298.2-484.4)$ & $500.3(393.1-577.3)$ & $0.019 * *$ \\
\hline & 2 & $264.8(145.5-463.7)$ & $142.9(93.7-195.5)$ & $176.9(103.4-405.9)$ & $0.019 * *$ \\
\hline & 3 & $1219.5(909.6-1494.0)$ & $1218.0(915.6-1422.0)$ & $1448.5(941.2-1699.0)$ & $0.572 * *$ \\
\hline
\end{tabular}

*ANOVA test, **Kruskal Wallis test; CRP - C-reactive protein, MCP-1 - monocyte chemoattractant protein-1, hsTnT - high sensitive troponin $\mathrm{T}, \mathrm{N}$ - total number of patients per group. The results are presented as median (IQR).

were tested using the Fisher's exact test and the chi square test. The association between the inflammation, stress and cardiac necrosis markers was tested using the Spearman's correlation analysis. The results of the 
correlation analysis were interpreted as follows: rho value from 0 to 0.25 or from 0 to -0.25 indicated the absence of correlation; rho value from 0.25 to 0.50 or from -0.25 to -0.50 was regarded as poor correlation between variables; rho value ranging from 0.50 to 0.75 or -0.50 to -0.75 pointed at moderate to good correlation; while rho from 0.75 to 1 or from -0.75 to -1 indicated very good to excellent correlation between the investigated variables (Udovicic et al. 2007).

Stepwise logistic and multiple regression analysis were used to investigate whether changes in certain markers can predict patient outcome. The differences in cortisol, CRP, leukocytes, IL-6, IL-10, MCP-1, resistin, leptin and hsTnT between the samples No. 1 and 2 and samples No. 2 and 3 were considered as independent variables. In the logistic regression the following outcomes were considered as dependent variables: use of ionotropic agents or vasopressors, electrocardiographic changes, atrial fibrillation, recurrent angina, postoperative myocardial infarction, stroke, kidney dysfunction, deep sternal bone infections and reoperation; while the time spent in the intensive unit, time to hospital discharge, duration of mechanical ventilation and blood loss in $\mathrm{ml}$ were considered as dependent variables in the multiple regression analysis.

All statistical analyses were performed with the MedCalc software, version 16.8 (Ostend, Belgium). Values of $\mathrm{P}<0.05$ were considered statistically significant. a)

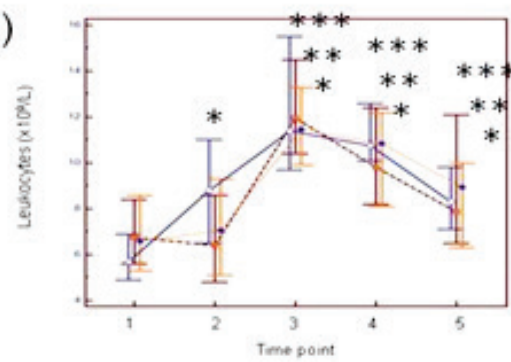

c)

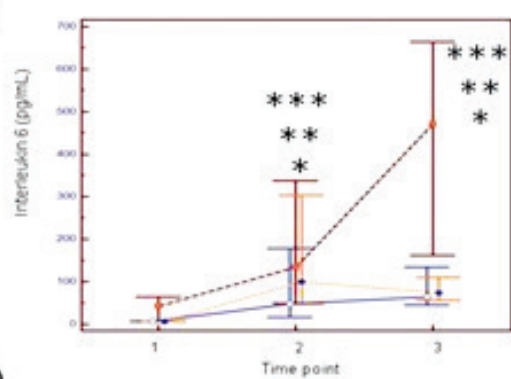

e)

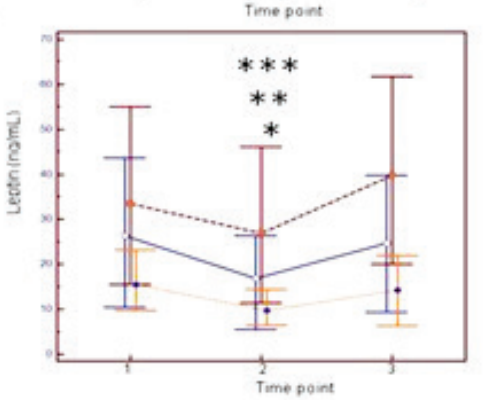

g)

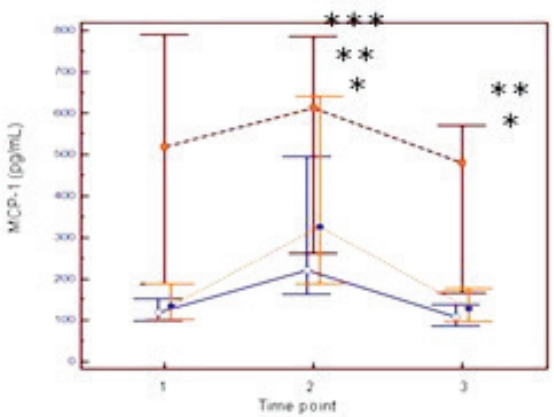

b)

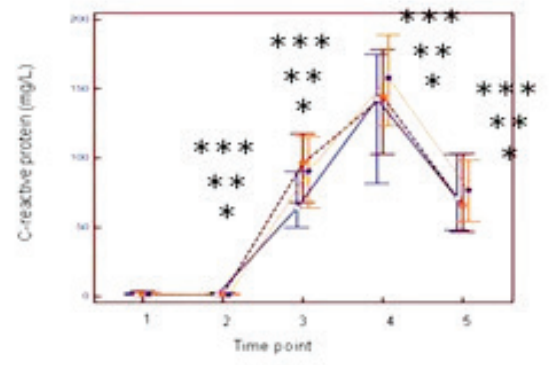

d)

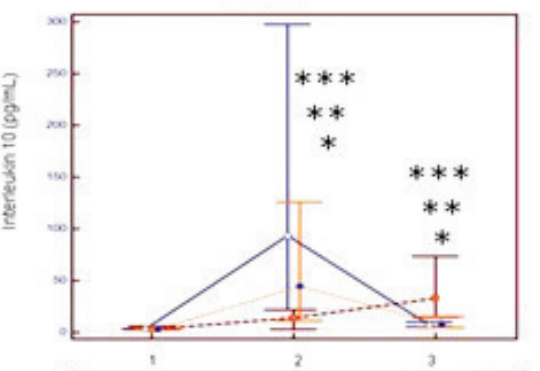

f)

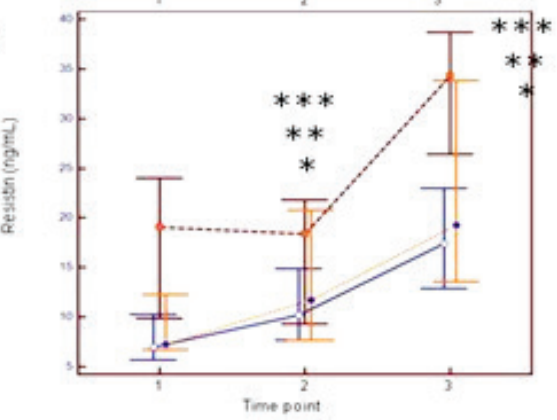

Fig. 1. The dynamic of inflammation markers (a) leukocytes, b) C-reactive protein, c) interleukin 6, d) interleukin 10, e) leptin, f) resistin and $\mathbf{g}$ ) monocyte chemo-attractant protein-1) during the perioperative surgery. *,**, and $* * *$ indicate statistically significant differences from the baseline value for AVRS, off-pump and on-pump group, respectively, as tested by Wilcoxon and paired t-test, as appropriate 

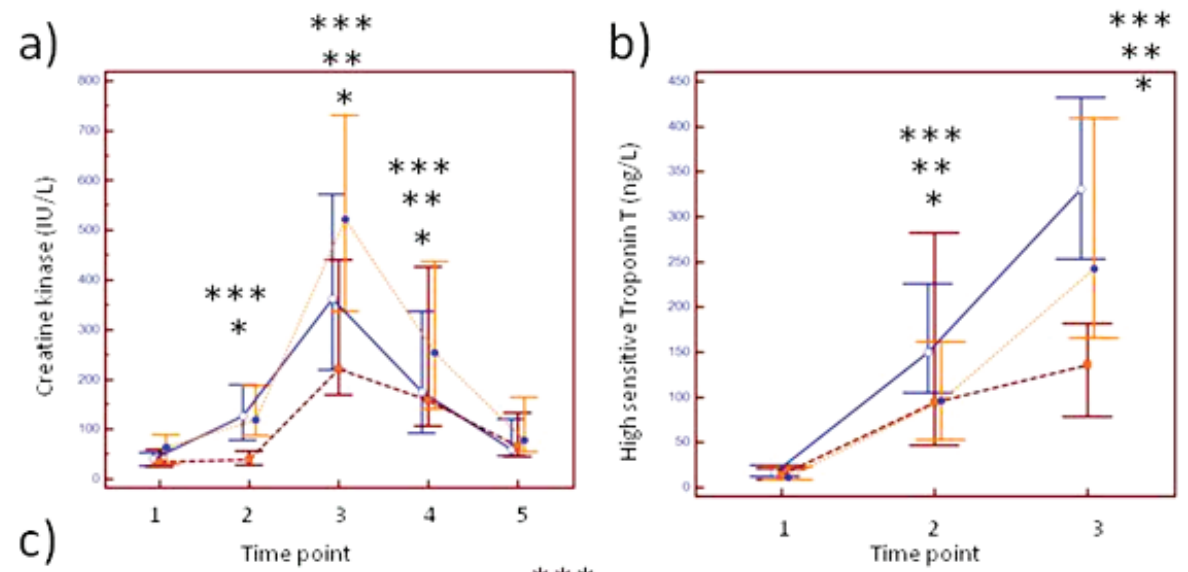

Fig. 2. The dynamic of cardiac and stress markers (a) creatine kinase, b) high sensitive Troponin $T$ and c) cortisol) during the perioperative surgery. $*, * *$, and $* * *$ indicate statistically significant differences from the baseline value for AVRS, off-pump and on-pump group, respectively, as tested.
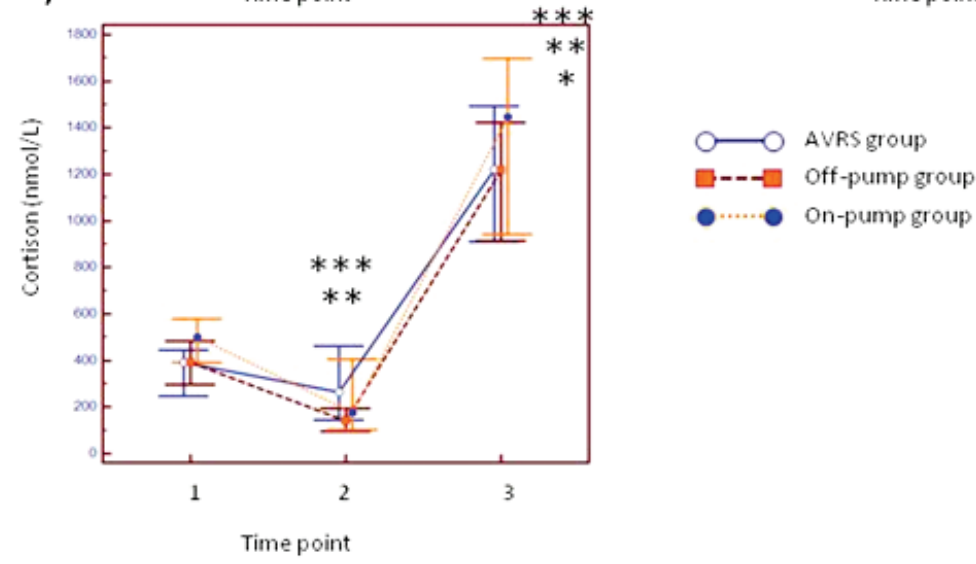

Table 5. Results of the logistic regression analysis

\begin{tabular}{|c|c|c|}
\hline Parameter & Odds ratio (95 \% CI) & $\mathbf{P}$ \\
\hline \multicolumn{3}{|c|}{ Transfusion needs } \\
\hline$\triangle R E S_{2-3}$ & $1.007(1.001-1.012)$ & 0.013 \\
\hline \multicolumn{3}{|c|}{ Use of inotropic agents or vasopressors } \\
\hline$\triangle C R P_{1-2}$ & $0.039(0.003-0.574)$ & 0.018 \\
\hline$\triangle R E S_{1-2}$ & $1.022(1.008-1.037)$ & 0.003 \\
\hline$\Delta$ Leuko $_{2-3}$ & $0.993(0.987-0.999)$ & 0.036 \\
\hline
\end{tabular}

$\triangle \mathrm{CRP}_{1-2}$ - difference in CRP before and at the end of the surgery; $\triangle \mathrm{RES}_{1-2}$ - difference in resistin before and at the end of the surgery; $\triangle \mathrm{RES}_{2-3}$ - difference in resistin at the end and 24 hours after the surgery; $\Delta \mathrm{LeukO}_{2-3}$ - difference in leukocytes count at the end and 24 hours after the surgery;

\section{Results}

Patient characteristics are presented in Table 3. The on-pump group of patients had more diabetic patients; more anastomoses made during the surgery, more patients with atrial fibrillation after surgery and lost more blood through drainage system after surgery than the other two groups of patients.

The results of tested markers at different time points are presented in Table 4. and the dynamics of them are shown in Figures 1 and 2.
Post hoc analysis showed significantly lower IL-10 levels immediately after the surgery and significantly higher levels of leptin and MCP-1 in the off-pump group than in the on-pump group. Off-pump group had significantly higher values of I-L6, IL-10, leptin, resistin and MCP-1 and significantly lower levels of CK and $h s \mathrm{TnT} 24$ after surgery than the on-pump group.

No or only weak correlation was found between stress, inflammation and cardiac necrosis markers. The only association that was moderate or very good was 
found between MCP-1 and resistin (Spearman's rho 0.754 (95\% CI $0.649-0.832), 0.820 \quad$ (95\% CI $0.738-0.878$ ) and 0.659 (95\% CI 0.523-0.762) for time points 1, 2 and 3, respectively).

Out of all regression analyses the investigated variables significantly predicted transfusion needs and the use of inotropic agents or vasopressors. The results are presented in Table 5 . The difference between resistin at the end and 24 hours after the surgery has shown small, but statistically significant contribution to predicting transfusion needs; while the difference between CRP and resistin before and at the end of the surgery together with the difference between leukocytes at the end and 24 hours after the surgery predicted the use of inotropic agents or vasopressors.

\section{Discussion}

Our results have confirmed the biphasic response of leptin, as previously shown in other studies, and the existence of significant correlation between MCP-1 and resistin. Additionally, this is the first study to investigate the changes in resistin concentration in patients undergoing valve replacement surgery in comparison with patients undergoing revascularization procedures. Resistin increased in all three investigated groups. However, the highest concentrations were found in the off-pump group.

The results of our study show that, out of the three investigated open heart surgical procedures, off-pump revascularization procedure was the one that caused the least adverse consequences. Namely, patients who underwent off-pump surgery spent less time on mechanical ventilation and fewer days in hospital before discharge, loss less blood, needed less transfusions, inotropic agents/vasopressors and had less cardiac and other adverse outcomes than patients undergoing valve replacement or on-pump revascularization surgery. Such favorable results are expected, since the operation is performed on a beating heart with continuous blood flow through the myocardium, except for the temporary ischemia around the places of anastomoses at the time of surgical suturing, followed by the reperfusion injury following clamp release at the treated area. To investigate what happens at the molecular level during heart surgery, we have sampled blood directly from coronary sinus just before the end of the procedure. Ischemia-reperfusion injury during surgical procedures induces the expression many cytokines, including IL-6, MCP-1 and leptin that act as warning signals for tissue damage and enhance monocytes/macrophages and $\mathrm{T}$ lymphocytes recruitment to the affected site (Tanaka et al. 2014, Deshmane et al. 2009, Matsui et al. 2007). These tissue infiltrated macrophages, among other, secrete resistin, a proinflammatory adipokine that further upregulates the expression of proinflammatory cytokines, thus enhancing the release of IL-6 and MCP-1 (Bokarewa et al. 2005). On one hand, our results support such thesis, since we found moderate and very good association between MCP-1 and resistin in all investigated time points. Correlation between resistin and MCP-1 has been previously demonstrated by an independent study recruiting participants without diabetes mellitus $(\mathrm{N}=123)$ and without known CVD or CVD risks (Aquilante et al. 2008). On the other hand, even though, earlier reports present that resistin upregulates IL-6 expression in addition to MCP-1, we did not confirm such correlation in our study. This is consistent with the results from Laurikka and co-workers who also did not find correlation between the mentioned parameters (Laurikka et al. 2014).

In our study, patients undergoing off-pump revascularization had higher concentrations off all four mentioned cytokines (IL-6, MCP-1, leptin and resistin) after the surgery when compared to the AVRS and on-pump revascularization group. Even though cardiopulmonary bypass induces global heart ischemia and consequently causes larger tissue injury it is possible that cardioplegic arrest that halts cellular metabolism and signal transduction could cause lower concentration of these markers in the procedures utilizing extracorporeal circulation. In addition, literature reports show that secreted leptin, MCP-1 and recruited macrophages as a result of ischemia reperfusion injury could exhibit cardioprotective, antiapoptotic effect on cardiomyocites and stimulate tissue regeneration (Frangogiannis 2015, Feijóo-Bandín et al. 2015, Morimoto and Takahashi 2007). Our finding would confirm these statements since the concentrations of cardiac necrosis markers (CK and $h s \operatorname{TnT}$ ) were the lowest in the off-pump revascularization group that also had the highest concentration of the mentioned markers.

Nevertheless, we have showed the same kinetics of leptin as other investigators (Hoda et al. 2006, Laurikka et al. 2014, Kremen et al. 2006). The transient decrease in leptin concentration was previously explained by the use of medications during surgery as well as fasting before and during the procedure. However, out of 
these studies only Hoda and his team compared leptin kinetics between different types of cardiac surgery. They found that leptin concentrations were lower in the off-pump group compared with the on-pump and heart valve replacement group what is contradictory to our results; however they recruited less participants per group ( $\mathrm{N}=19$ for on-pump, $\mathrm{N}=15$ for heart valve replacement and $\mathrm{N}=15$ for off-pump group) what could have contributed to these differences. Additionally, Hoda's team found a moderate correlation (Pearson's rho was $0.43, \mathrm{P}<0.02)$ between serum leptin and cortisol during the perioperative period. In our study, cortisol kinetics showed similar behavior as leptin (decrease immediately after the surgery followed by increase 24 hours postoperatively); however, there was no correlation between the two parameters at either investigated time point. In addition to Hoda, Kain and his team also found moderate correlation between plasma leptin and cortisol $(\mathrm{r}=0.40, \mathrm{P}$ value not presented) only on female patients $(\mathrm{N}=21)$ undergoing total abdominal hysterectomy (Kain et al. 1999). Investigations on pediatric population undergoing congenital heart defects $(\mathrm{N}=29)$ showed inverse correlation of leptin and cortisol $(\mathrm{r}=-2.8, \mathrm{P}<0.01)$ (Modan-Moses et al. 2001). Despite the different correlation between leptin and cortisol, which could be due to the divergences in the studied populations and number of recruited participants, all the mentioned studies showed decrease at the end of the surgery followed by an increase in leptin concentration during the perioperative period of open-heart surgery.

Several studies have shown an increase in resistin concentration during the perioperative period in patients elective for on-pump revascularization and valve replacement surgery (Laurikka et al. 2014, Németh et al. 2014, Kremen et al. 2006). In addition, Németh and co-workers, who presented their results as a poster, found no differences in plasma resistin concentration in patients undergoing of-pump coronary artery bypass what is contradictory to our results, since we found higher concentrations in off-pump group when compared to on-pump and AVRS groups. Since they have presented their results only as a poster abstract, the only reasons for which we can argue to have contributed to divergent results are the number of recruited patients (16 on-pump and 19 off-pump versus 30 patients in each group in our study) and possibly method of resistin determination (ELISA vs. multiplex determination by Luminex ${ }^{\circledR}$ ). Other data regarding patient characteristics, operating techniques are unfortunately unavailable at the time of manuscript writing.

All of the so far mentioned cytokines play very important proinflammatory roles in the cardiac injury during the perioperative period, however their actions are tightly regulated by anti inflammatory molecules that control the intensity of the immune reaction. In this study we have investigated IL-10 and confirmed previously reported higher concentrations of IL-10 in on-pump compared to off-pump surgery at the end of the operation. Additionally, our results show that among the three studied groups, the patients in the AVRS group had the highest IL-10 concentration at the end of the surgery. The higher concentrations of IL-10 both in the on-pump and AVRS group could be explained by the immuneinhibiting reaction of the organism towards the exposure of blood to extracorporeal circulation. Interestingly, though the IL-10 concentration decreased after surgery in the AVRS and the on-pump groups, it continued to increase in the off-pump group. Our results are similar to those found by Castellheim and co-workers (Castellheim et al. 2008). They have recruited 22 patients undergoing off-pump and 22 patients undergoing on-pump revascularization surgery and took samples at 7 different time points during and after the surgery (T1 - after induction of general anesthesia and before systemic heparinization; T2 - immediately after institution of extracorporeal circuit or $30 \mathrm{~min}$ after $\mathrm{T} 1$ in the off-pump group; T3 - $45 \mathrm{~min}$ after T2; T4 - $60 \mathrm{~min}$ after $\mathrm{T} 2$; $\mathrm{T} 4$ - 90 min after $\mathrm{T} 2 ; \mathrm{T} 6$ - at termination of the cardiopulmonary bypass or $120 \mathrm{~min}$ after $\mathrm{T} 2$ in the off-pump group; T7 - during skin closure; T8 $=$ two hours after the surgery). As of their results, IL-10 concentration during skin closure was higher in the on-pump than in the off-pump group. After 2 hours, its concentration began to increase in the off-pump group, while started decreasing in the on-pump group. This could probably explained by a slightly delayed immune response due to the elimination of artificial extracorporeal circuit stimulus.

In addition to investigating the changes of inflammatory response, stress and cardiac markers we wanted to examine whether intensity of changes in a certain marker can predict short-term patient outcome. We found that the change in resistin concentration at the end and 24 hours after the surgery has shown small, but statistically significant contribution to predicting transfusion needs; while the difference between CRP and resistin before and at the end of the surgery together with the difference between leukocytes at the end and 24 hours after the surgery predicted the use of inotropic agents or 
vasopressors. Although, the change in CRP concentration has shown statistically the lowest OR value, the presented change in CRP before and after the surgery was clinically insignificant (median values for 1 st and 2 nd sampling point were 1.0 and $1.1 ; 1.6$ and $1.4 ; 1.5$ and $1.0 \mathrm{mg} / \mathrm{l}$ for AVRS, off-pump and on-pump group, respectively). In the literature, we were able to find only one study that investigated the association of resistin with outcomes following lower extremity revascularization (Owens et al. 2010). Owens and colleagues followed 225 patients undergoing lower extremity bypass surgery and found that resistin (hazard ratio (HR) 1.75, $95 \% \mathrm{CI}, 1.07-2.85$; $\mathrm{P}=0.025$ ) and CRP (HR 2.39, $95 \%$ CI, 1.30-4.39; $\mathrm{P}=0.005$ ) were independently predictive of reduced amputation-free survival. Since the values of our odds ratios are close to 1 , it would be of benefit to conduct a study on an independent patient population to confirm whether these markers can serve as predictors of shortterm outcomes.

The immune reaction during surgical procedures is very complex due to many contributing factors that include tissue injury, different extents of ischemiareperfusion injury and exposure to artificial surfaces of the extracorporeal circuit which depends on the operating technique. Also, concentration of certain markers, such as leptin, might show different kinetics depending on gender and/or presence of certain diseases (i.e. diabetes mellitus) which could be a potential limitation to our study. As mentioned before, many studies gained divergent result regarding the kinetics of, for example of IL-6 and correlation between different parameters; therefore it would be useful to conduct a study that would recruit a larger number of patients. Additionally, we would like to point out that this study did not aim to find an ideal biomarker that will solve all of the problems with predicting patient outcome after cardiac surgery, but to find association between certain molecules and perioperative conditions. An ideal biomarker would be the one that is highly specific for the condition of interest, has a highly accurate receiver operating characteristic curve, contributes significantly to the patient outcome, has a fast turnaround time, is easily available and cheap. However, finding such clinically ideal markers is not an easy nor simple process. In such a quest, the results from studies such as ours and similar ones contribute to the body of knowledge and enable scientists and clinicians to single out markers that could potentially have greater value in diagnostics and patient follow up.

Nevertheless, our study is the first one to report the kinetics of adipokine resistin in patients undergoing aortic valve replacement surgery and compared its kinetics with other types of open-heart operating techniques. In addition, our study population is homogenous with no concomitant operations and conversions from one operating technique to another, which could have introduced a certain bias to our results.

To conclude, open heart surgery causes an increase of inflammatory, stress and cardiac markers. Concentrations of IL-6, leptin, resistin and MCP-1 were the highest in off-pump groups which could resulted in cardioprotection by lower $\mathrm{CK}$ and $h s \mathrm{TnT}$ concentrations. Out of the investigated molecules only resistin concentration correlates with the release of MCP-1 which confirms the link between resistin secreted form infiltrated macrophages and enhanced release of MCP-1. Additional research is needed to confirm whether changes in resistin, leukocytes and CRP during the perioperative period can predict the needs for transfusion and the use of inotropes/vasopressors.

\section{Conflict of Interest}

There is no conflict of interest.

\section{Acknowledgements}

This study was supported by the Ministry of Science, Education and Sports, Republic of Croatia (project number: 134-1340227-0200).

\section{References}

AQUILANTE CL, KOSMISKI LA, KNUTSEN SD, ZINEH I: Relationship between plasma resistin concentrations, inflammatory chemokines, and components of the metabolic syndrome in adults. Metabolism 57: 494-501, 2008. https://doi.org/10.1016/j.metabol.2007.11.010

ASGEIRSSON T, ZHANG S, KHOO SK, RESAU JH, DUJOVNY N, SENAGORE AJ: Serum Adiponectin, Resistin, and Circulating Soluble Receptor for Advanced Glycation End Products in Colectomy Patients. Mediators Inflamm 2011: 916807, 2011. https://doi.org/10.1155/2011/916807 
BIGLIOLI P, CANNATA A, ALAMANNI F, NALIATO M, PORQUEDDU M, ZANOBINI M, TREMOLI E, PAROLARI A: Biological effects of off-pump vs. on-pump coronary artery surgery: focus on inflammation, hemostasis and oxidative stress. Eur J Cardiothorac Surg 24: 260-269, 2003. https://doi.org/10.1016/S10107940(03)00295-1

BOKAREWA M, NAGAEV I, DAHLBERG L, SMITH U, TARKOWSKI A: Resistin, an adipokine with potent proinflammatory properties. J Immunol 174: 5789-5795, 2005. https://doi.org/10.4049/jimmunol.174.9.5789

CASTELlHEIM A, HOEL TN, VIDEM V, FOSSE E, PHARO A, SVENNEVIG JL, FIANE AE, MOLLNES TE: Biomarker profile in off-pump and on-pump coronary artery bypass grafting surgery in low-risk patients. Ann Thorac Surg 84: 1994-2002, 2008. https://doi.org/10.1016/j.athoracsur.2008.03.012

CUNDRLE Jr. I, SUK P, SRAMEK V, LACINOVA Z, HALUZIK: Circadian leptin concentration changes in critically ill heart failure patients. Physiol Res 67: 505-508, 2018. https://doi.org/10.33549/physiolres. 933712

DESHMANE SL, KREMLEV S, AMINI S, SAWAYA BE: Monocyte Chemoattractant Protein-1 (MCP-1): An overview. J Interferon Cytokine Res 29: 313-326, 2009. https://doi.org/10.1089/jir.2008.0027

DIEGELER A, DOLL N, RAUCH T, HABERER D, WALTHER T, FALK V, GUMMERT J, AUTSCHBACH R, MOHR FW: Humoral immune response during coronary artery bypass grafting: A comparison of limited approach, "off-pump" technique, and conventional cardiopulmonary bypass. Circulation 102: III95-100, 2000. https://doi.org/10.1161/01.CIR.102.suppl_3.III-95

European Cardiovascular Disease Statistics, 2017 edition. Available at: http://www.ehnheart.org/cvd-statistics/cvdstatistics-2017.html. Accessed: 25 July 2018.

FEIJÓO-BANDÍN S, PORTOLÉS M, ROSELLÓ-LLETÍ E, RIVERA M, GONZÁLEZ-JUANATEY JR, LAGO F: 20 years of leptin: Role of leptin in cardiomyocyte physiology and physiopathology. Life Sci 140: 10-18, 2015. https://doi.org/10.1016/j.lfs.2015.02.016

FRANGOGIANNIS NG: Emerging roles for macrophages in cardiac injury: cytoprotection, repair and regeneration. J Clin Invest 125: 2927-2930, 2015. https://doi.org/10.1172/JCI83191

FRANKENSTEIN L, WU AH, HALLERMAYER K, WIANS FH JR, GIANNITSIS E, KATUS HA: Biological variation and reference change value of high-sensitivity troponin $\mathrm{T}$ in healthy individuals during short and $\begin{array}{llllll}\text { intermediate follow-up periods. Clin } \quad \text { Chem } & \text { 57: }\end{array}$ https://doi.org/10.1373/clinchem.2010.158964

GIBBISON B, SPIGA F, WALKER JJ, RUSSELL GM, STEVENSON K, KERSHAW Y, ZHAO Z, HENLEY D, ANGELINI GD, LIGHTMAN SL: Dynamic pituitary-adrenal interactions in response to cardiac surgery. Crit Care Med 43: 791-800, 2015. https://doi.org/10.1097/CCM.0000000000000773

HODA MR, EL-ACHKAR H, SCHMITZ E, SCHEFFOLD T, VETTER HO, DE SIMONE R: Systemic stress hormone response in patients undergoing open heart surgery with or without cardiopulmonary bypass. Ann Thorac Surg 82: 2179-2186, 2006. https://doi.org/10.1016/j.athoracsur.2006.06.087

KAIN ZN, ZIMOLO Z, HENINGER G: Leptin and the perioperative neuroendocrinological stress response. J Clin Endocrinol Metab 84: 2438-2442, 1999. https://doi.org/10.1210/jc.84.7.2438

KAWAHITO K, ADACHI H, INO T: Influence of surgical procedures on interleukin 6 and monocyte chemotacting and activating factor responses: CABG vs. Valvular surgery. J Interferon Cytokines Res 20: 1-6, 2000.

https://doi.org/10.1089/107999000312676

KNUDSEN LS, CHRISTENSEN IJ, LOTTENBURGER T, SVENDSEN MN, NIELSEN HJ, NIELSEN L, HØRSLEV-PETERSEN K, JENSEN JE, KOLLERUP G, JOHANSEN JS: Pre-analytical and biological variability in circulating interleukin 6 in healthy subjects and patients with rheumatoid arthritis. Biomarkers 13: 59-78, 2008. https://doi.org/10.1080/13547500701615017

KREMEN J, DOLINKOVA M, KRAJICKOVA J, BLAHA J, ANDERLOVA K, LACINOVA Z, HALUZIKOVA D, BOSANSKA L, VOKURKA M, SVACINA S, HALUZIK M: Increased subcutaneous and epicardial adipose tissue production of proinflammatory cytokines in cardiac surgery patients: possible role of insulin resistance. J Clin Endocrinol Metab 91: 4620-4627, 2006. https://doi.org/10.1210/jc.2006-1044

LAURIKKA A, VUOLTEENAHO K, TOIKKANEN V, RINNE T, LEPPÄNEN T, TARKKA M, LAURIKKA J, MOILANEN E: Adypocitokines resistin correlates with oxidative stress and myocardial injury in patients undergoing cardiac surgery. Eur J Cardiothorac Surg 46: 729-736, 2014. https://doi.org/10.1093/ejcts/ezt634 
MATLOCH Z, KOTULAK T, MALUZIK M: The role of epicardial adipose tissue in heart disease. Physiol Res 65: 23-32, 2016. https://doi.org/10.33549/physiolres.933036

MATSUI H, MOTOOKA M, KOIKE H, INOUE M, IWASAKI T, SUZUKI T, KURABAYASHI M, YOKOYAMA T: Ischemia/reperfusion in rat heart induces leptin and leptin receptor gene expression. Life Sci 80: 672-680, 2007. https://doi.org/10.1016/j.lfs.2006.10.027

MENG F, MA J, WANG W, LIN B: Meta-analysis of interleukin 6, 8 and 10 between off-pump and on-pump coronary artery bypass groups. Bosn J Basic Med Sci 17: 85-94, 2017. https://doi.org/10.17305/bjbms.2017.1505

MODAN-MOSES D, EHRLICH S, KANETY H, DAGAN O, PARIENTE C, ESRAHI N, LOTAN D, VISHNE T, BARZILAY Z, PARET G: Circulating leptin and the perioperative neuroendocrinological stress response after pediatric cardiac surgery. Crit Care Med 29: 2377-2382, 2001. https://doi.org/10.1097/00003246-200112000$\underline{00022}$

MODAN-MOSES D, KANETY H, DAGAN O, PARIENTE C, BEN-ABRAHAM R, FREEDMAN L, PRINCE T, SHIMON I, BARZILAY Z, PARET G: Circulating leptin levels after cardiopulmonary bypass in children. J Cardiothorac Vasc Anesth 15: 740-744, 2011. https://doi.org/10.1053/jcan.2001.28320

MORIMOTO H. TAKAHASHI M: Role of monocyte chemoattractant protein-1 in myocardial infarction. Int J Bio Sci 3: 159-166, 2007.

NÉMETH Á, CZIRÁKI A, SULYOK E, HORVÁTH IG, ALOTTI N, RAUH M, RASCHER W, SZABADOS S: Short-term response of metabolic hormones to coronary artery bypass surgery. Adv Med Sci 59: 213-220, 2014. https://doi.org/10.1016/j.advms.2014.04.002

ONORATI F, RUBINO AS, NUCERA S, FOTI D, SICA V, SANTINI F, GULLETTA E, RENZULLI A: Off-pump coronary artery bypass surgery versus standard linear or pulsatile cardiopulmonary bypass: endothelial activation and inflammatory response. Eur J Cardiothorac Surg 37: 897-904, 2010. https://doi.org/10.1016/j.ejcts.2009.11.010

OWENS CD, KIM JM, HEVELONE ND, HAMDAN A, RAFFETTO JD, CREAGER MA, CONTE MS: Novel adipokines, high molecular weight adiponectin and resistin, are associated with outcomes following lower extremity revascularization with autogenous vein. J Vasc Surg 51: 1152-1159, 2010. https://doi.org/10.1016/j.jvs.2009.12.051

SERRANO-MARTÍNEZ M, PALACIOS M, LEZAUN R: Monocyte chemoattractant protein-1 concentration in coronary sinus blood and severity of coronary disease. Circulation 108: e75, 2003. https://doi.org/10.1161/01.CIR.0000089100.20182.B7

TANAKA T, NARAZAKI M, KISHIMOTO T: IL-6 in inflammation, immunity, and disease. Cold Spring Harb Perspect Biol 6: a016295, 2014. https://doi.org/10.1101/cshperspect.a016295

UDOVICIC M, BAZDARIC K, BILIC-ZULLE L, PETROVECKI M: What we need to know when calculating the coefficient of correlation? Biochem Med (Zagreb) 17: 10-15, 2007. https://doi.org/10.11613/BM.2007.002

WEHLIN L, VEDIN J, VAAGE J, LUNDAHL J: Peripheral blood monocyte activation during coronary artery bypass grafting with or without cardiopulmonary bypass. Scand Cardiovasc J 39: 78-86, 2005. https://doi.org/10.1080/14017430410004623 